\title{
Computed tomographic colonography: Diagnosis of an incarcerated Spigelian hernia
}

\begin{abstract}
Author:
Joel H. Bortz ${ }^{1}$

Affiliation:

${ }^{1}$ LSG Imaging, Los Angeles,

United States of America

Correspondence to:

Joel Bortz

Email:

joelbortzmd@gmail.com

Postal address:

13031 Villosa Place,

Apartment 141, Playa Vista,

California 90094,

United States of America

Dates:

Received: 19 June 2014

Accepted: 27 July 2014

Published: 04 Dec. 2014

How to cite this article:

Bortz, JH. Computed

tomographic colonography:

Diagnosis of an incarcerated

Spigelian hernia. S Afr J

Rad. 2014;18(1); Art. \#673,

4 pages. http://dx.doi.

org/10.4102/sajr.v18i1.673

\section{Copyright:}

(C) 2014. The Authors.

Licensee: AOSIS

OpenJournals. This work is

licensed under the Creative

Commons Attribution

License.
\end{abstract}

Read online:
A 68-year-old obese man underwent computed tomographic colonography (CTC) scanning to investigate worsening constipation and lower abdominal discomfort on his left side. Optical colonoscopy was contraindicated because of his comorbidities. A preliminary CTC diagnosis of incarcerated Spigelian hernia was made, based on lateral deviation of the sigmoid colon to the left as well as extrinsic impressions on it, and the central location of the small bowel. Spigelian hernia is a rare form of anterior abdominal wall hernia. CTC can play a role in its diagnosis.

\section{Introduction}

Computed tomography (CT) has been shown to produce the most accurate imaging in the diagnosis of Spigelian hernia. In this case report, CT colonography (CTC) demonstrated additional findings, making the diagnosis more readily achievable.

\section{Case report}

A 68-year-old obese man was referred for CTC scanning by a gastroenterologist. His primary clinical history was worsening constipation and lower abdominal discomfort on his left side. Optical colonoscopy was contraindicated because of his comorbidities of hypertension, ischaemic heart disease, diabetes and chronic obstructive airways disease. A low-dose CTC technique, $\mathrm{CO}_{2}$ insufflation and a 16-slice General Electric multidetector CT (MDCT) scanner were used for the study. As the patient was obese, a standard two-view supine and prone study could not be performed; therefore right and left decubitus views were done instead. Filling the colon with three litres of $\mathrm{CO}_{2}$ took approximately seven minutes, compared with the average time of three minutes.

The CTC study showed an incarcerated Spigelian hernia (SH), without evidence of obstruction. The colon map views showed three important findings: (1) the sigmoid colon was markedly displaced to the left; (2) the small bowel was in a central position (Figure 1a); and (3) in addition, the air-contrast colon map demonstrated two external impressions on the sigmoid colon at the point where it enters and exits the muscular defect (Figure 1b). Slight narrowing of both the afferent and efferent loops of the sigmoid was noted on 3D fly-through imaging (Figure 2).

A 2D image below the umbilicus confirmed a defect through both the internal oblique and the transversus muscles. The sigmoid colon was contained in a hernia. An axial view demonstrated a thin rim of the external oblique muscle laterally, indicating an interstitial or interparietal geographic location of the hernia (Figure 3). Coronal and sagittal 2D images clearly demonstrated the $\mathrm{SH}$ (Figure 4 and Figure 5).

In view of the patient's comorbidities, surgical intervention was not an option at this stage. He was fully briefed by the gastroenterologist in this regard and made aware of the risk of obstruction or strangulation. He was informed that, should this occur, surgical intervention would be required.

\section{Discussion}

Spigelian hernia is also known as spontaneous anterior abdominal hernia. It is a rare acquired hernia occurring in less than $2 \%$ of all abdominal hernias, with just over 1000 reported cases in the literature. ${ }^{1}$ Congenital cases of $\mathrm{SH}$ have been reported in children. ${ }^{2} \mathrm{SH}$ is often undetected clinically as the symptoms and physical findings are subtle or inconclusive, particularly in obese adult patients. $\mathrm{SH}$ is prone to complications, such as incarceration, small or large bowel obstruction and strangulation, because the hernia defect is usually narrow $(2 \mathrm{~cm}-3 \mathrm{~cm}) .^{1-3}$ 

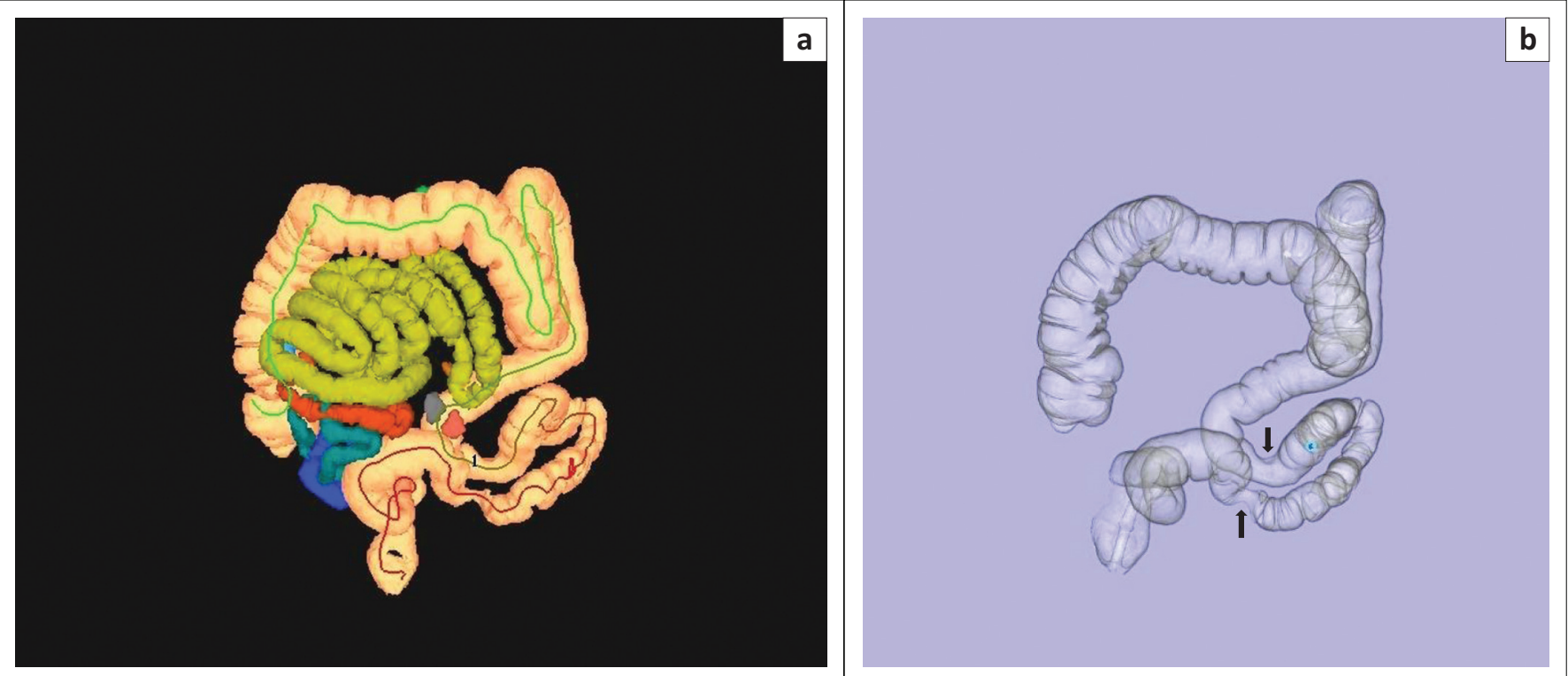

FIGURE 1: Colon map showing small bowel (light green) in central positon. Note that the sigmoid colon is markedly displaced to the left; (b) Colon map using air-contrast indicates locus of narrowing of the sigmoid loops (arrows).

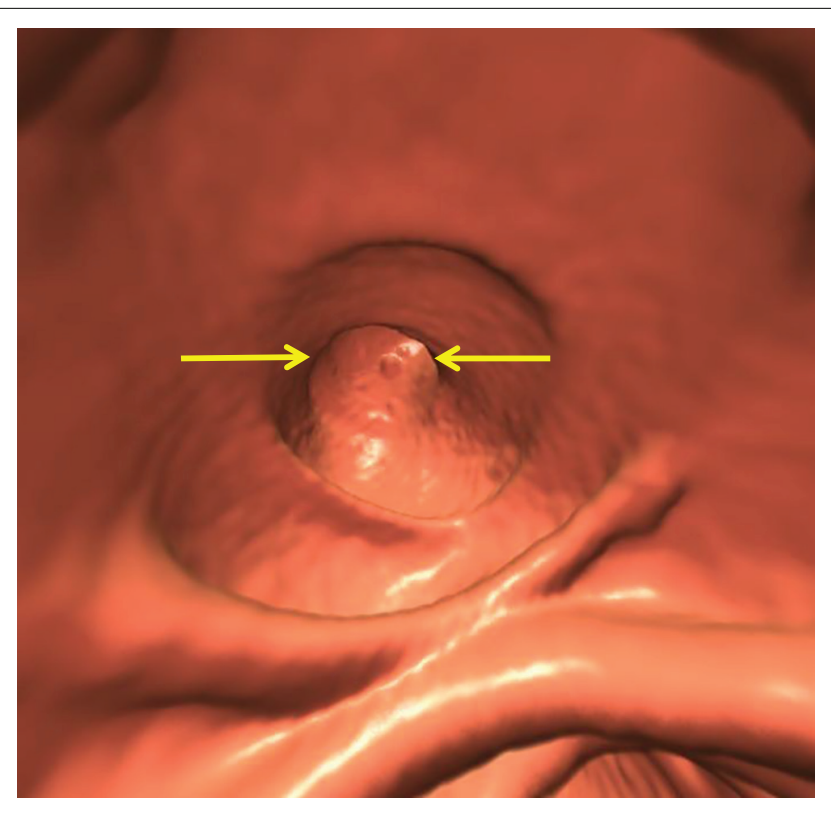

FIGURE 2: A 3D fly-through image shows narrowing of efferent loop of sigmoid colon (arrows).

Diagnosis is, however, primarily made by $\mathrm{CT}^{1,4,5}$ In the present case report, a CTC was performed for investigation of abdominal discomfort and worsening constipation. A diagnosis of $\mathrm{SH}$ was made based on the lateral deviation of the sigmoid colon as well as the two extrinsic defects on its wall (Figure 1a and Figure 1b). The diagnosis was confirmed by the 2D study which showed an interparietal $\mathrm{SH}$ containing sigmoid colon and a clear hernial defect in both the internal oblique and transversus muscles (Figure 4 and Figure 5). CTC is a minimally invasive, fast, safe and accurate screening examination for colorectal cancer. It is also suitable as a diagnostic study for patients with gastrointestinal symptoms. Furthermore, it allows evaluation of extracolonic structures in the abdomen and pelvis.

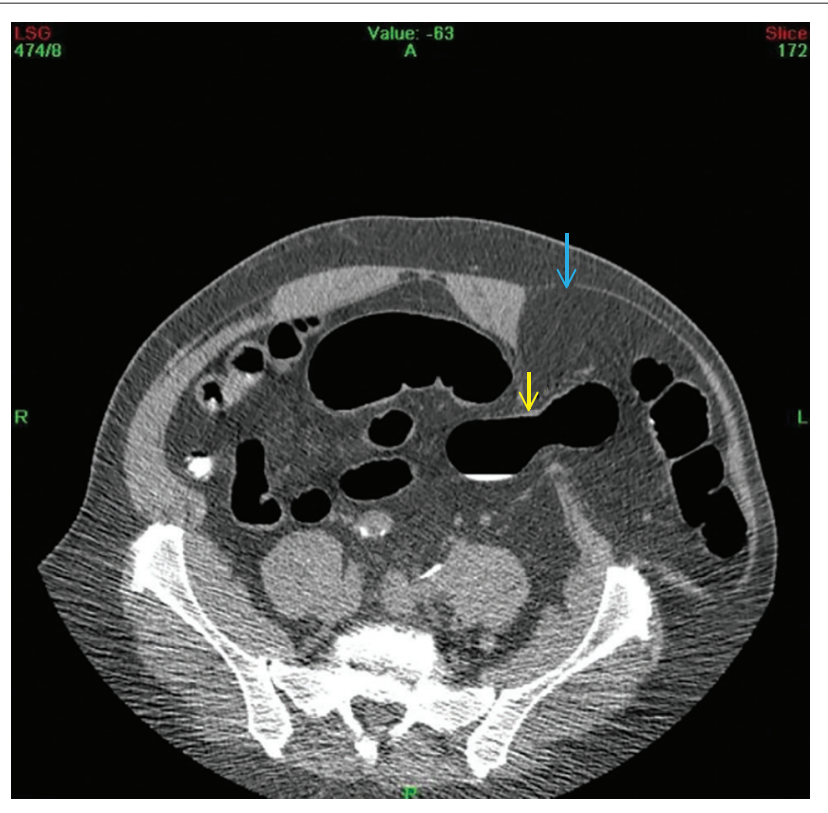

FIGURE 3: Axial view shows the sigmoid colon passing through the hernial defect (yellow arrow). Blue arrow shows the thin rim of the external oblique muscle.

Ultrasound is often the first line of imaging used to investigate SH. ${ }^{1,2,5}$ Although operator dependent, it can be used to make a diagnosis of $\mathrm{SH}$ in a large proportion of patients. In addition, a Valsalva manoeuvre can be used in supine and erect sonography to determine the presence of $\mathrm{SH}^{3}$

There is a slightly higher incidence of $\mathrm{SH}$ in women than in men; in adults, it usually occurs during the fourth and seventh decades. ${ }^{1,2,6}$ It may occur on either side of the body, or occasionally bilaterally. ${ }^{1}$ There are several known predisposing factors: obesity, chronic obstructive airways disease, previous surgery or laparoscopy, peritoneal dialysis, multiple pregnancies, and rapid weight loss, for example..$^{2,6,7}$ 


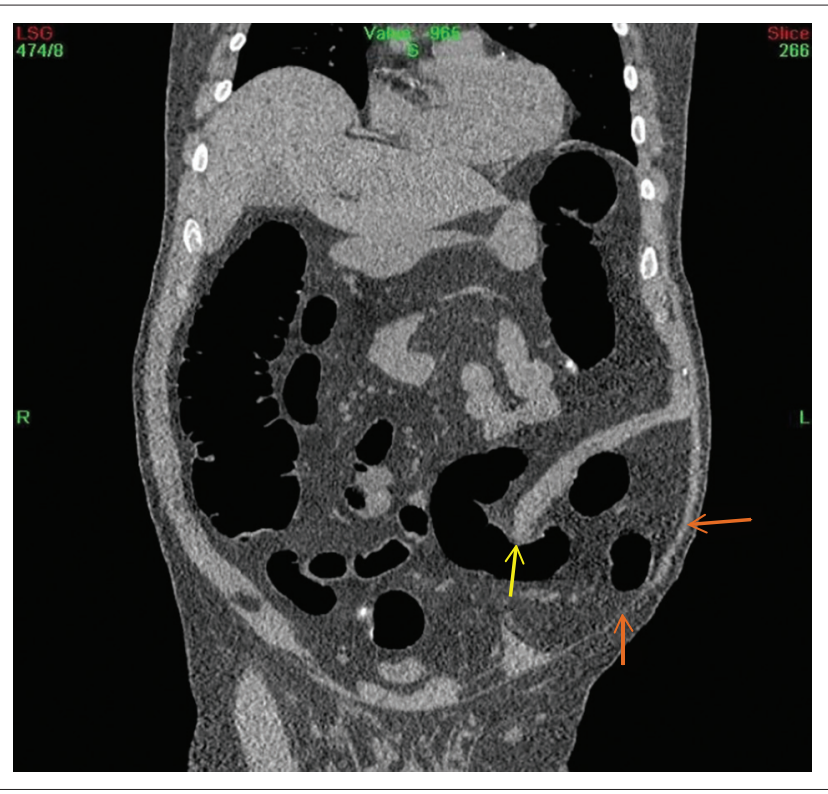

FIGURE 4: Coronal 2D image of Spigelian hernia through the muscular defect. The hernia is contained by the external oblique muscle laterally, and shows marked thinning inferiorly (orange arrows). Yellow arrow indicates site of muscular defect.

Clinical presentation varies, depending on the content of the hernial sac. In obese individuals, detection of SH may be particularly difficult as the hernia may not be palpable on clinical examination. Some patients may complain of pain, but no lump is palpable. ${ }^{6}$ Omentum and/or segments of the small bowel or colon are usually present in the sac. In the present case, the colon map showed small bowel in a central location; it was not included in the hernia sac (Figure 1a). Other structures, such as the stomach, Meckel's diverticulum, the appendix, and testicular and endometrial tissue, have been found in $\mathrm{SH}^{2,8}$

It is important to know the anatomy of the abdominal musculature when diagnosing $\mathrm{SH}$. There are three flat and two vertical muscles of the anterior abdominal wall (Figure 6a and Figure $6 \mathrm{~b}$ ). The flat group consists of the external and internal oblique muscles and the transversus abdominis muscles. The vertical group comprises the rectus abdominis and pyramidalis.

The external oblique muscle arises from the lower eight ribs; it is the largest and most superficial muscle of the antero-lateral abdominal wall. The muscle fibres pass inferomedially. The aponeurosis of the upper part of the muscle splits to cover the anterior and posterior portions of the rectus abdominis. Inferiorly, the superior margin of the external oblique aponeurosis thickens and folds back on it to form the inguinal ligament. ${ }^{9}$

The internal oblique is the middle of the flat muscle group. It is a thin muscular sheet that fans out antero-medially. Its fibres become aponeurotic and split to envelop the anterior and posterior margins of the rectus in its upper two-thirds of the abdomen. In the lower third, the aponeurosis does not split; it passes as a whole in front of the rectus to reach the linear alba (Figure 6a). ${ }^{9}$

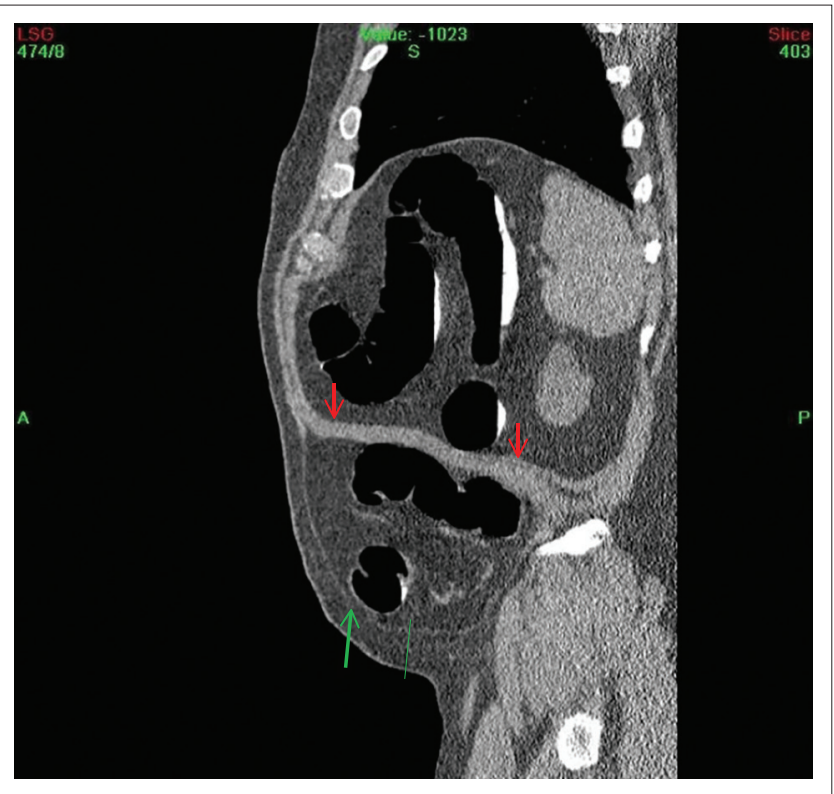

FIGURE 5: Sagittal 2D image shows the Spigelian hernia sandwiched between (1) the internal oblique muscle and transversus muscle superiorly (red arrows) and (2) thinned-out external oblique muscle inferiorly (green arrow).

The transversus abdominis muscle is the innermost of the three flat muscles, with a transversomedial orientation. Superior to the arcuate line, the aponeurosis of the tranversus abdominis muscle splits to form part of both the anterior and posterior rectus sheath. Inferiorly to the arcuate line, the aponeurosis of the transversus abdominis muscle passes anteriorly to form part of the anterior rectus sheath layer. This arrangement allows for a potential area of weakness posteriorly.

The rectus abdominis is a vertical muscle that is wrapped anteriorly and posteriorly by the aponeurosis of the three flat muscles. The lower third has no posterior cover. The pyramidalis is absent in approximately $25 \%$ of individuals. It is a small, insignificant muscle situated in relation to the inferior margin of the rectus.

The Spigelian (semilunar) line represents the vertical fibrous band formed by the union of the rectus sheath with the fascia of the three flat muscles of the anterior abdominal wall. In the upper two-thirds of the abdomen, the aponeurosis splits at the lateral margin of the rectus into a posterior layer, which passes behind the rectus muscle, and an anterior layer which passes in front of the rectus. The level at which this occurs is known as the arcuate line (Figure 6a), the semicircular line or the line of Douglas. The deep layer of the abdominal wall is only weakly reinforced by separated fascial bands. The Spigelian aponeurosis is the portion of the transversus abdominis that is bordered by the semilunar (Spigelian) line laterally and the lateral borders of the rectus muscle medially (Figure 6a). SH protrudes through this aponeurosis; most are found in a transverse band lying between $0 \mathrm{~cm}$ and $6 \mathrm{~cm}$ cranially to a line running between both the anterior and superior iliac spines. ${ }^{8}$ This band is referred to as the 'Spigelian hernia belt' where the Spigelian fascia is widest (Figure 6a). ${ }^{6}$ 


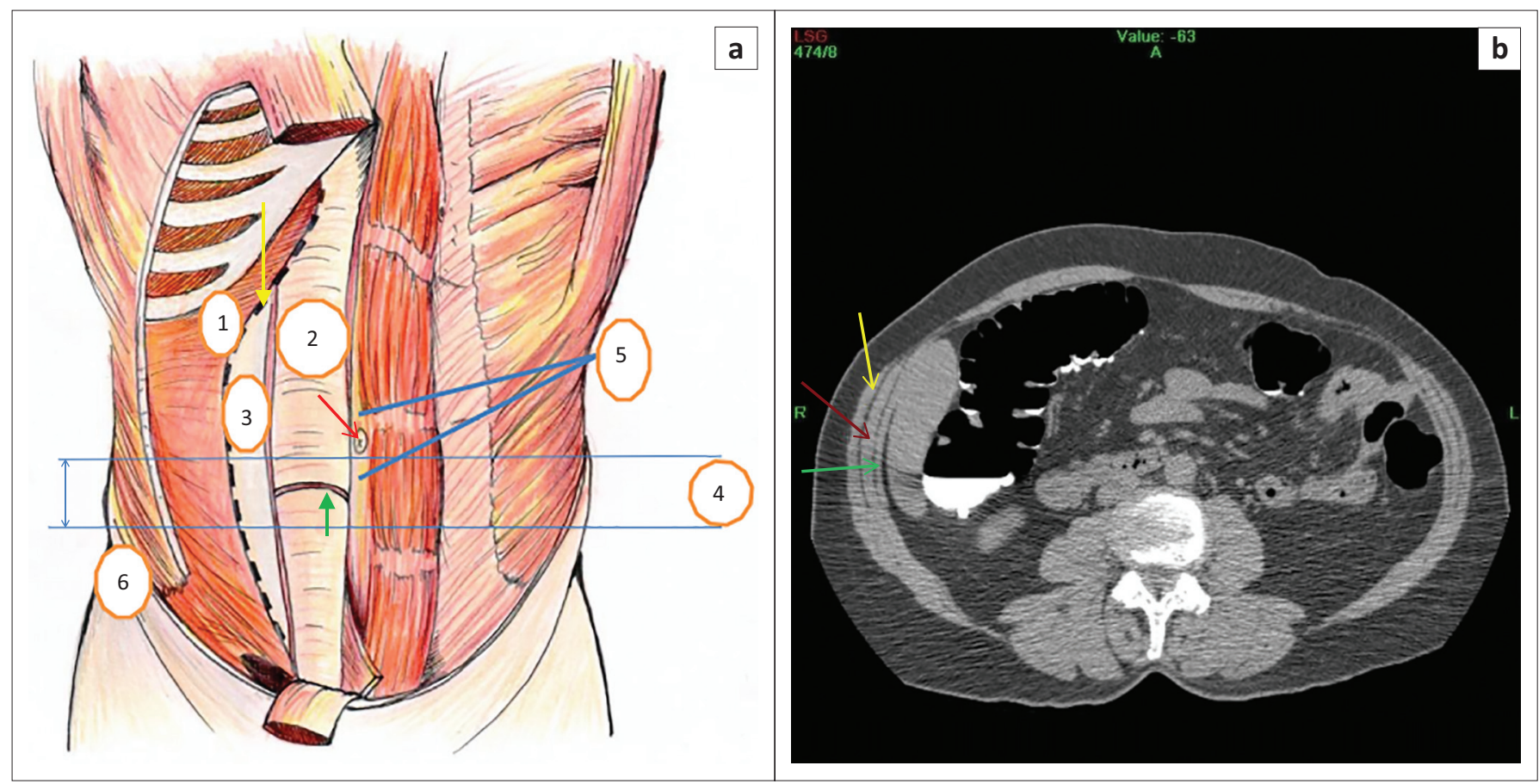

FIGURE 6: (a) View of the anterior abdominal wall with the external oblique, internal oblique and rectus abdominis muscles peeled away on the right side of the body (adaptation with grateful acknowledgement). ${ }^{2}$ Identified are (1) the transversus muscle; (2) the posterior rectus sheath; (3) the Spigelean aponeurosis; (4) the Spigelian hernia belt (5) the linear alba; and (6) the anterior superior iliac spine. Yellow arrow = semilunar line; red arrow = umbilicus; green arrow = arcuate line; and doublepointed blue arrow $=6 \mathrm{~cm}$ width of the Spigelian hernia belt. (b) Axial CT image shows the three flat abdominal muscles: yellow arrow $=$ external oblique; red arrow $=$ internal oblique; and green arrow = abdominis transversus.

A diagnosis of $\mathrm{SH}$, based on plain-film radiographs, upper and lower gastrointestinal studies, and follow-through studies, is not readily made in the absence of an intestinal obstruction. ${ }^{7}$ There has been reported use of magnetic resonance imaging (MRI) for investigation of $\mathrm{SH} .{ }^{10} \mathrm{CT}$, however, is the main imaging modality. As is evident in the present case report, CTC can play an important part in the diagnosis of $\mathrm{SH}$. The main advantage of CTC is that the entire large bowel and all extracolonic components can be visualised. The complementary role of $2 \mathrm{D}$ and $3 \mathrm{D}$ imaging in the diagnosis of $\mathrm{SH}$ is well demonstrated in this case report.

\section{Acknowledgements}

Clinton Bopp is thanked for drawing the line diagram of the abdominal musculature.

\section{Competing interests}

The author declares that he had no financial or personal relationship(s) that might have inappropriately influenced him in writing this report.

\section{References}

1. Moles Morenilla L, Docobo Durántez F, Mena Robles J, de Quinta Frutos R. Spigelian hernia in Spain. An analysis of 162 cases. Rev Esp Enferm Dig. 2005;97:338-347. http://dx.doi.org/10.4321/S1130-01082005000500006

2. Salameh JR. Primary and unusual abdominal wall hernias. Surg Clin N Am. 2008;88:45-60. http://dx.doi.org/10.1016/j.suc.2007.10.004

3. Mariolis-Sapsakos T, Kalles V, Papapanagiotou I, et al. A rare case of Spigelian hernia penetrating the external oblique muscle. OA Case Reports. 2013;28;2(2):11.

4. Emby DJ, Aoun G. CT technique for suspected anterior abdominal wall hernia. AJR. 2003;181(2):431-433. http://dx.doi.org/10.2214/ajr.181.2.1810431

5. Balthazar EJ, Subramanyam BR, Megibow A. Spigelian hernia: CT and ultrasonography diagnosis. Gastrointest Radiol. 1984;9(1):81-84. http:// dx.doi.org/10.1007/BF01887808

6. Mittal T, Kumar V, Khullar R, et al. Diagnosis and management of Spigelian hernia: A review of literature and our experience. J Minim Access Surg. 2008;4(4):95-98. $\mathrm{http}: / / \mathrm{dx}$.doi.org/10.4103/0972-9941.45204

7. Ribeiro EA, Cruz RJ, Moreira SM. Intestinal obstruction induced by a giant incarcerated Spigelian hernia. Case report and review of the literature. Sao Paulo Med J. 2005;123(3):148-150. http://dx.doi.org/10.1590/S151631802005000300012

8. Angelici AM, Nasti AG, Petrucciani N, Leonetti G, Palumbo P. Spigelian hernia: A case report and review of the literature. G Chir. 2006;27(11-12):433-435.

9. Netter F. The Ciba collection of medical illustrations. Vol 3. Digestive system. Part 2. Lower digestive tract. New York: Colour Press, 1962; p. 12-14.

10. Kassir R, Tarantino E, Lacheze R, Brek A, Bartolmeo AD, Tiffet O. Management of Spigelian hernia caused by necrobiotic fibroma of the uterus in a pregnant woman. Int J Surg Case Rep. 2013;4(12):1176-1178. http://dx.doi.org/10.1016/j.ijscr.2013.10.010 